\title{
A Method for Rapid Measurement of Contrast Sensitivity on Mobile Touch-Screens
}

\author{
Jeffrey B. Mulligan; NASA Ames Research Center; Moffett Field, CA/USA
}

\begin{abstract}
Touch-screen displays in cell phones and tablet computers are now pervasive, making them an attractive option for vision testing outside of the laboratory or clinic. Here we describe a novel method in which subjects use a finger swipe to indicate the transition from visible to invisible on a grating which is swept in both contrast and frequency. Because a single image can be swiped in about a second, it is practical to use a series of images to zoom in on particular ranges of contrast or frequency, both to increase the accuracy of the measurements and to obtain an estimate of the reliability of the subject. Sensitivities to chromatic and spatio-temporal modulations are easily measured using the same method. A prototype has been developed for Apple Computer's iPad/iPod/iPhone family of devices, implemented using an open-source scripting environment known as QuIP (QUick Image Processing, http://hsi.arc.nasa.gov/groups/scanpath/research.php). Preliminary data show good agreement with estimates obtained from traditional psychophysical methods as well as newer rapid estimation techniques. Issues relating to device calibration are also discussed.
\end{abstract}

\section{Introduction}

Reports of vision impairment following long-duration space flight have been hypothesized to be due to elevated intracranial pressure, resulting from fluid shifts in response to microgravity; Vision Impairment and Intracranial Pressure (VIIP) is now a topic of active research $[1,2,3,4,5,6,7]$. This work was motivated by the idea that a method for performing a quick assessment of vision in a minute or two could enable weekly or even daily monitoring of astronaut vision. Mobile devices such as Apple Computer's iPod Touch have the additional desirable feature of low mass. Simple, portable tools for vision assessment may also have clinical applications on earth.

While Snellen acuity is still the most common clinical description of a person's vision (e.g., "20/20"), contrast sensitivity is a richer and more informative descriptor. The contrast sensitivity function (CSF) describes the sensitivity to sinusoidal grating stimuli, where "sensitivity" is defined as the reciprocal of the threshold contrast [8]. Acuity measured with full-contrast letters is roughly equivalent to specifying the high-frequency cut-off of the CSF: both answer the question of what is the smallest thing that can be seen when presented at high contrast. Different ocular pathologies affect the CSF in different ways. A focus error will manifest primarily as a degradation at high frequencies, while a cataract produces a loss of sensitivity at all frequencies.

Traditionally, the measurement of contrast sensitivity has been a somewhat laborious task, requiring presentation of multiple stimuli above and below threshold at each frequency of in- terest. A number of charts designed for clinical use have been developed to provide a fast and easy method to estimate contrast sensitivity in a clinical setting $[9,10]$. More recently, the "Quick CSF" method has been introduced to allow estimation of the form of the CSF as a whole, without time-consuming measurement of the sensitivity at individual frequencies [11, 12]. The Uppsala Contrast Sensitivity Test (UCST) also provides a characterization of contrast sensitivity in 10-20 seconds [13].

The image shown in figure 1 shows an image designed to allow a person to visualize their own CSF. The image is a sinusoidal grating that is swept in frequency in one dimension, while a linear variation of contrast is applied in the orthogonal dimension. The bars corresponding to frequencies of high sensitivity (usually around 1 cycle per degree) will appear to extend further than those corresponding to frequencies of low sensitivity. Thus the region where visible stripes are seen is bounded by the CSF.

The contrast/frequency sweep image is the basis of the proposed method. Such an image is presented on a touch screen, and the subject is instructed to swipe their finger along the boundary between the regions containing visible and invisible pattern. It is an easy task which requires little training; the primary problem arises when subjects agonize over what to do, "over-thinking" the task.

The sweep image was first produced in 1964 by Fergus Campbell and John Robson, who made a photographic time exposure of an oscilloscope screen [14]. It was first shown to the public later that year at a meeting of the Optical Society of America, in Washington DC, where a small number of photographic prints were provided. A version of the image subsequently appeared in Floyd Ratliff's 1965 book [15]. It also appears in Cornsweet's 1970 book [16], attributed to an unpublished photograph provided by Fergus Campbell. A similar image appears in the optics literature considerably earlier [17], but these authors make no mention of the visual appearance of the pattern, perhaps because for testing lenses the test pattern was fabricated at a fine scale outside the range of normal unaided vision.

Campbell and Robson had the idea to have a subject mark the boundary of visibility,but concluded "that for several reasons this was not at all a satisfactory procedure" [18]. These reasons include the dependence of the percept upon where the subject is looking, and movements of the eyes; the presence of the boundary line (if drawn) can also affect the percept [14]. A slightly different procedure involving a sweep of contrast only was introduced by Arden [19], but, according to Robson, the technique "was unable to provide sufficiently reliable measurements" [18].

\section{Methods}

A prototype implementation has been developed for Apple Computer's iOS platform, supporting the iPad, iPod Touch, and 
iPhone. Table 1 gives display parameters for the devices tested.

\begin{tabular}{|l|l|l|l|l|}
\hline Device & size & resolution & pitch & S.F. range \\
\hline iPad 2 & 9.7 & $1024 \times 768$ & 132 & $0.09-11.5$ \\
\hline iPad 4 & 9.7 & $2048 \times 1536$ & 264 & $0.09-23$ \\
\hline iPad mini & 7.9 & $1024 \times 768$ & 163 & $0.11-14.2$ \\
\hline iPod 4 & 3.5 & $960 \times 640$ & 326 & $0.24-28.4$ \\
\hline iPod 5 & 4.0 & $1136 \times 640$ & 326 & $0.20-28.4$ \\
\hline
\end{tabular}

Table 1: Parameters of Apple touch-screen devices. Size column gives the screen diagonal in inches, pitch is specified in units of pixels per inch, and minimum and maximum spatial frequencies are specified in cycles per degree (assuming a viewing distance of 24 inches and a minimum period of 4 pixels).

\section{General methods}

The prototype application was developed as a set of scripts for the QuIP interpreter. QuIP is an open-source program developed in-house, and made freely available under the terms of the NASA Open Source Agreement (NOSA). "QuIP" is an acronym for "Quick Image Processing," and also captures the flavor of rapid prototyping with short scripts, or "quips." The system provides facilties for image processing and scientific computing comparable to commercial interactive systems such as Matlab, while providing tight coupling to the underlying hardware.

\section{Image rendering}

Generation of the low-contrast stimuli needed for the measurement of contrast threshold presents a technical challenge, given that the majority of digital displays offer only 8 bits of intensity resolution. We address the problem by using spatial dithering or halftoning to render the fine gradations that are lost by intensity quantization. To minimize visibility of the dither pattern, an iterative algorithm was used to minimize the error with respect to a vision model [20]. The entire process is illustrated in figure 1. The results appear to be sufficient to eliminate quantization artifacts, but additional improvements in luminance resolution could be achieved at the expense of high-frequency chromatic errors $[21,22,23]$, or temporal dithering [24, 25].

\section{Calibration}

Presentation of stimuli of known contrast (as in the measurement of contrast sensitivity) requires correction of nonlinearities relating pixel values to output luminances (gamma). In the laboratory, this is commonly done with a calibrated photometer, but to support the calibration of mobile devices in the field, it is desirable to have a method that does not depend on additional equipment. Here we present a psychophysical method, and compare the accuracy and reliability of the results with traditional photometer measurements.

\section{Photometer-based calibration}

Traditional photometer measurements were made for the purpose of comparison. Devices were operated in slave mode by a remote computer which collected photometer readings after setting the display output level. The measurement system consisted of a UDT photodiode, equipped with a photometric filter and "lumilens", connected to a transimpedance amplifier, the output of which was fed to a 16 bit analog-to-digital converter. Different
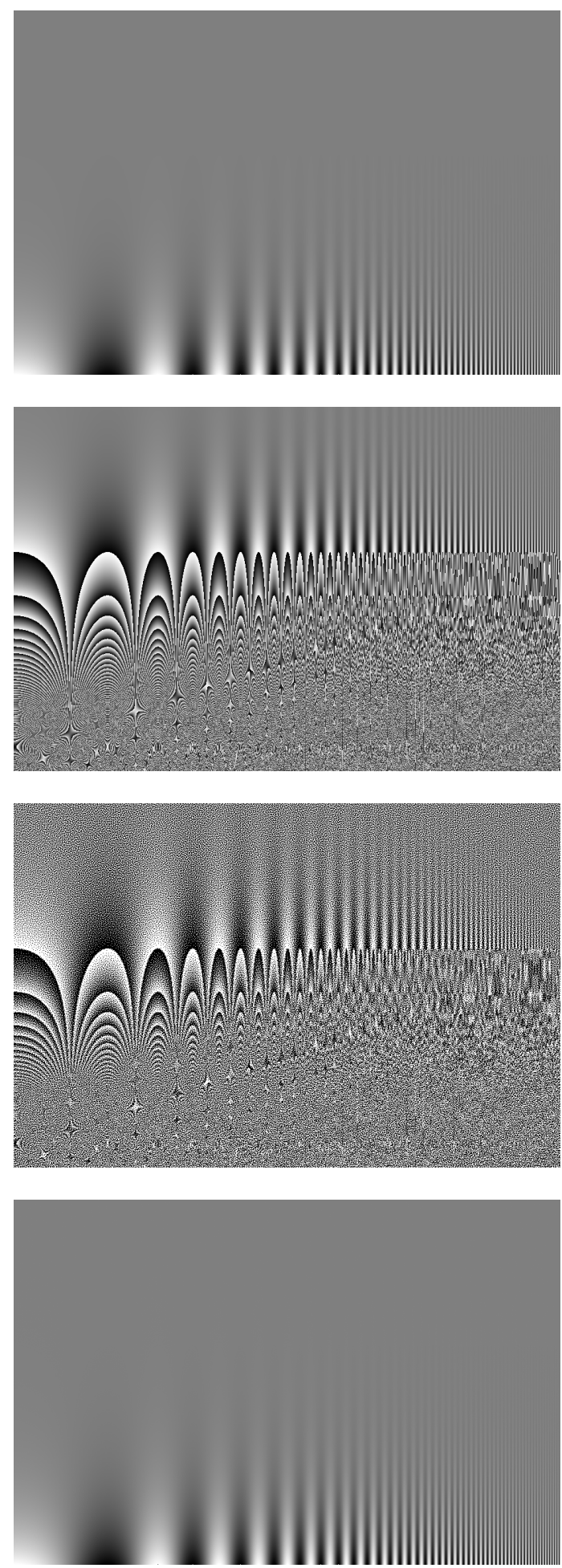

Figure 1: Steps in precision rendering of a sweep grating. Top panel: initial image, quantized to 8 bits. The horizontal line above which no modulation is present may be visible. Second from top: residual error, scaled up to full display range to aid visualization. Third from top: residual error converted to a one-bit image via model-based halftoning. Bottom image: final image produced by adding the halftone to the low-order bit of the quantized image. 


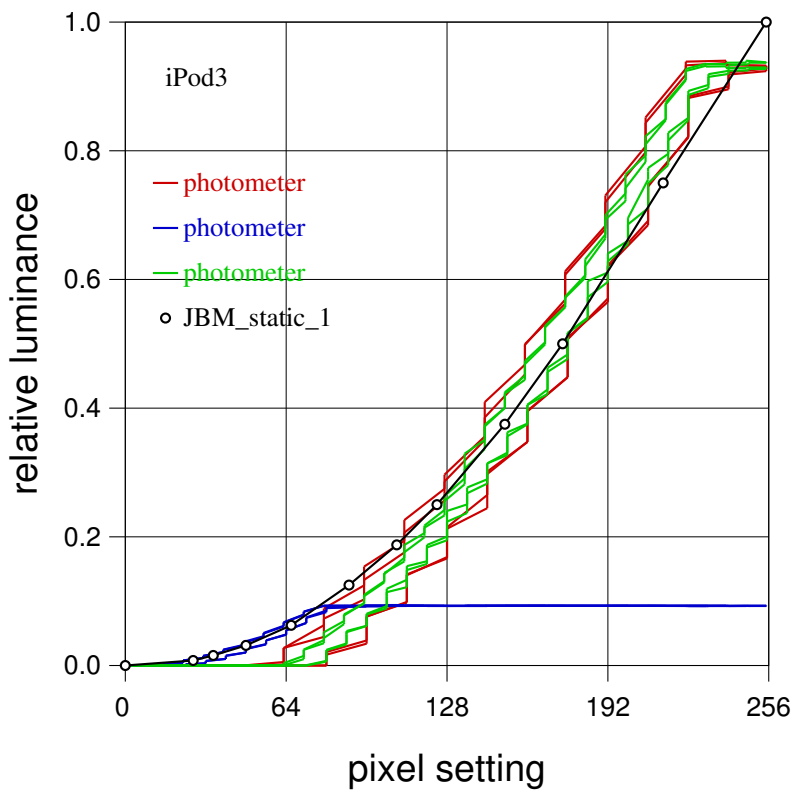

Figure 2: Photometer measurements showing hysteresis.

amplifier gain settings were needed to measure the high and low parts of the range.

Typical results are shown in figure 2. Data from three different measurement scans are shown. In each scan, the display setting was stepped from 0 to 255 and back to 0 again. The particular device (a 3rd generation iPad) demonstrates significant hysteresis: at a given setting, the readings are higher when measured in a descending sequence than in an ascending sequence. The blue curve plots results from a scan with the amplifier gain increased by a factor of 10 .

\section{Psychophysical calibration}

Two psychophysical methods for determining the display gamma have been evaluated, one based on static brightness matching, and another based on a motion-nulling task. The motion nulling task was inspired by the work of Anstis and Cavanagh [26], who used motion nulling to match the luminances of different colors. Here, we use motion nulling to match the luminances of spatial mixtures of different gray levels. The subject views a repetetive loop of four carefully-constructed frames, and adjusts a variable gray level to null the apparent motion, which happens when the variable luminance matches that of a stippled pattern made up of two fixed levels. Each iteration of the procedure results in finding the setting that bisects the two fixed settings, and the entire gamma curve is traced out by a process of recursive subdivision. Additional details of the procedure can be found in [27].

The patterns used to make the four frames of the motionnulling sequence were also displayed as four strips, one above the other, as shown below. At the motion null, the contrast in the first and third rows should be zero.

Two subjects repeated the calibration times three times each for both static and motion methods. It can be seen that there is a systematic difference between the two methods, with the static method requiring higher settings to match a given luminance. We
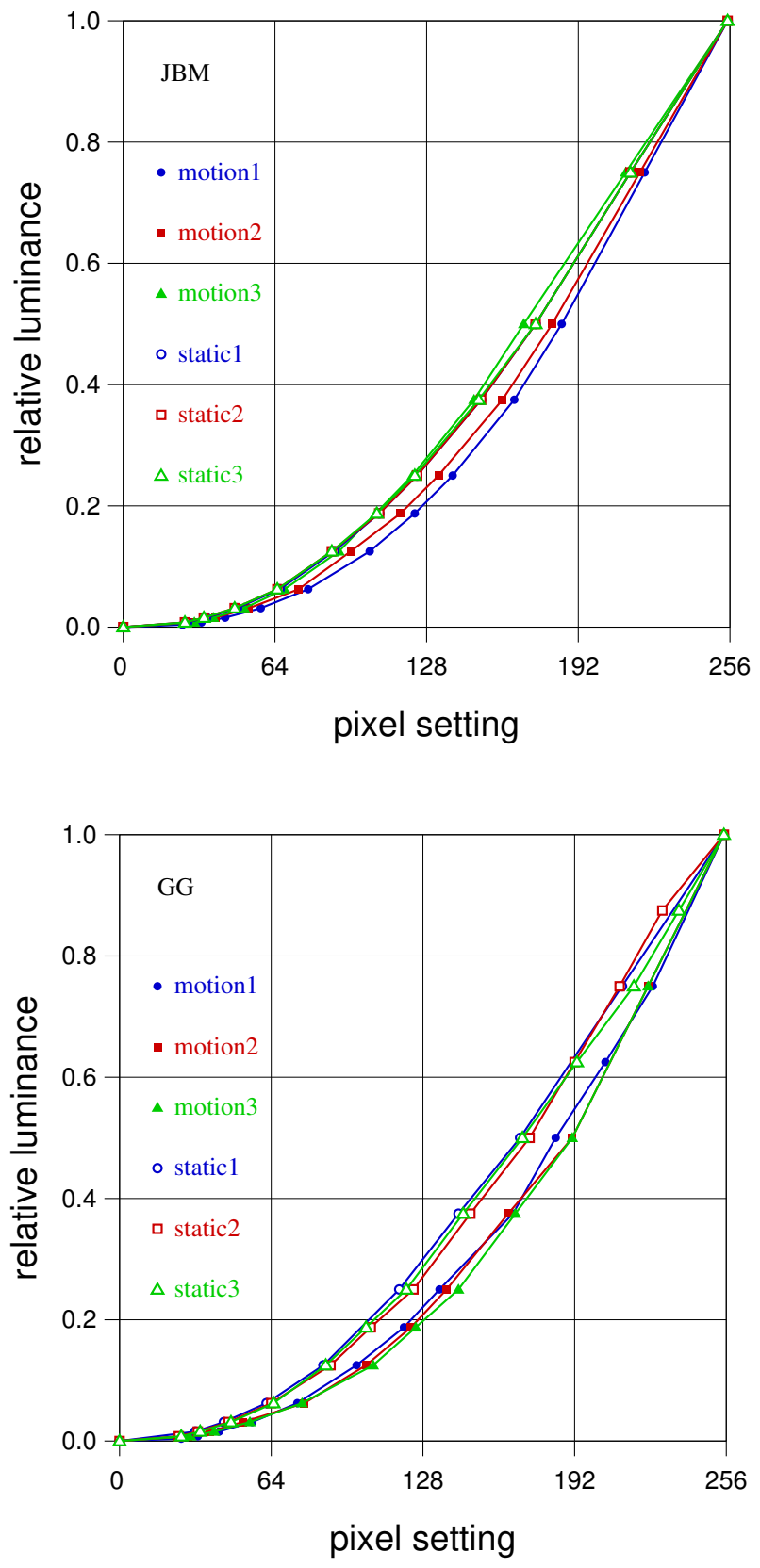

Figure 3: Estimated gamma functions estimated using the static method (filled symbols) and the motion method (open symbols), for subject JBM (upper panel) and GG (lower panel).

hypothesize that this results from an interaction of the hysteresis (seen in figure 2) with the sequence of patterns used to generate the motion stimulus; this will be tested by both repeating the procedure using a device with no hysteresis, and also by creating a new motion stimulus in which the spatial phase of the stipple patterns is changed on alternate frames (which may reverse the discrepancy).

\section{Camera-based calibration}

We have explored using an uncalibrated camera instead of a photometer for display calibration. Unfortunately, the exponential 
ambiguity [28] prevents joint calibration of display and camera nonlinearities using single pixel levels, but this can be overcome using dithered patterns, under the assumption of spatial independence, following the work of Olczak and Tumblin [29]. We generated full-field images consisting of a fine checkerboard of two pixel levels, and slightly defocussed the camera lens so that the pattern was not resolved. A two-dimension scan was then performed over pairs of values for the two pixel levels. For each pair of levels, a series of images was acquired from the camera (Point Grey Research, Flea3), and the camera pixel values from the relevant region were averaged to form a single reading. Figure 4 shows a set of such readings displayed graphically, where the intensity of each square is proportional to the corresponding reading.

From a tableau of measurements such as this, we can simultaneously solve for the pixel transmission and camera response functions. The pixel transmission function can be represented as the set of values (between 0 and 1) corresponding to the transmission at each setting. We used a parameterized function to represent the camera response:

$$
R(l)=R_{0}+\left(k_{C} l\right)^{\gamma}
$$

The parameter $R_{0}$ is an offset representing the response to no light, $k_{C}$ is a scale factor applied to relative luminance, and $\gamma$ is an exponent representing the "gamma" of the camera. The camera used has user-programmable gamma, which was set to a value of 1 .

We solved simultaneously for both the display and camera parameters using Levenberg-Marquardt optimization [30]. Because each camera measurement depends only on 2 of the $N$ display parameters, the Jacobian matrix relating the measurements to the parameters is sparse, allowing a computationally efficient version of the algorithm to be employed [31]. The estimate of the camera response arrived at by this method is shown in figure 5; the estimated value of the gamma parameter is 0.978 , fairly close to the nominal value of 1 . Figure 6 shows the estimate of the display response function (in red filled symbols), plotted along with one of the estimates from the static psychophysical method. The agreement is good, except for the low end of the curve, where a negative value of the parameter $R_{0}$ causes some of the values to dip below zero. As a negative luminance is physically impossible, it is apparent that one or more of the assumptions in the model has been violated. Nevertheless, the results compare favorably with those shown in figure 2, demonstrated that uncalibrated cameras can provide calibrations as good or better than dedicated photometers. In future work, we plan to explore using a mirror with the device's front-facing camera to perform automatic calibrations with a minimum of additional equipment.

\section{CSF estimation}

Following Lesmes et al. [11, 32], we have modelled the CSF as a parabola. Because of the range of spatial frequencies is limited at the low end by the small size of the mobile devices used, we have not incorporated the low-frequency plateau incorportated by Lesmes et al. The resulting fit can be characterized by three parameters, the most important of which are the peak sensitivity value, and the frequency of peak sensitivity.

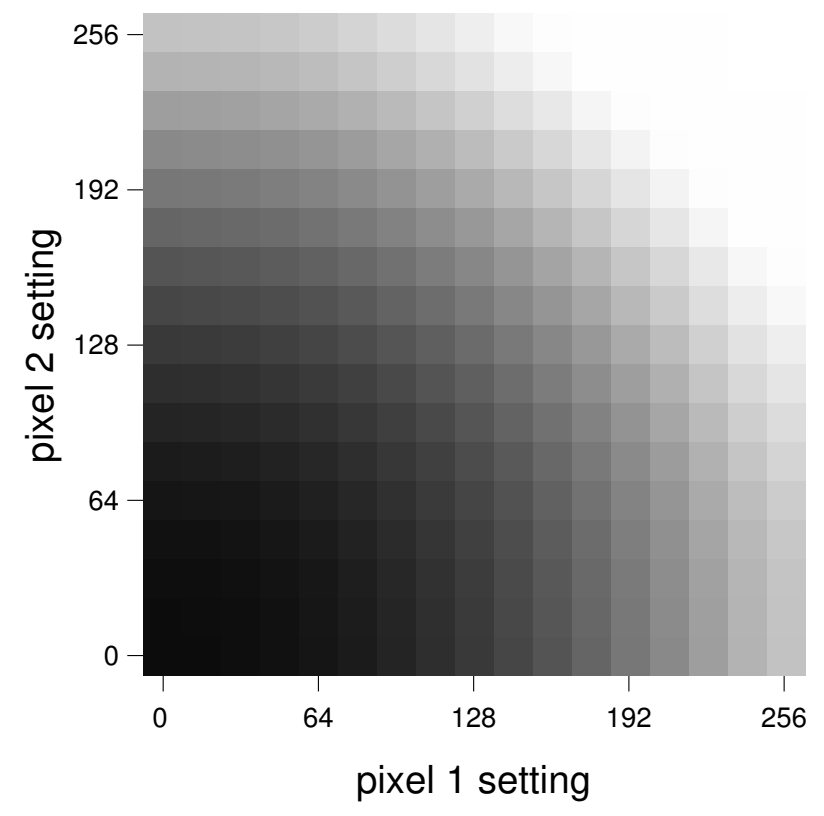

Figure 4: Tableau of camera measurements presented as an image.

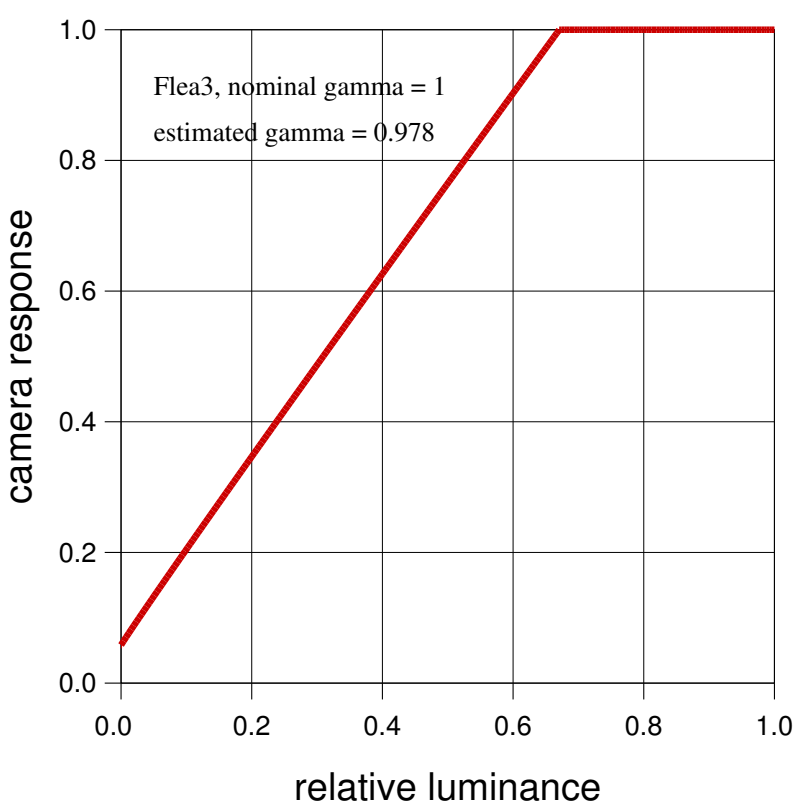

Figure 5: Camera response estimated from the data in figure 5.

\section{Results}

To obtain an estimate of the reliability of CSF estimates obtained with the swipe method, a subject performed 10 repetitions. For each incremental set of swipes, a parabola was fit to the data. Figure 7 shows the evolution of the parameter estimates as the data were collected. Also shown (in thin lines) are the estimates when the swipes are processed not in the order they were collected, but rather sorted on the value of the parameter estimate from each individual swipe; the upper line represents the estimates when the swipes are processed in descending order of the 


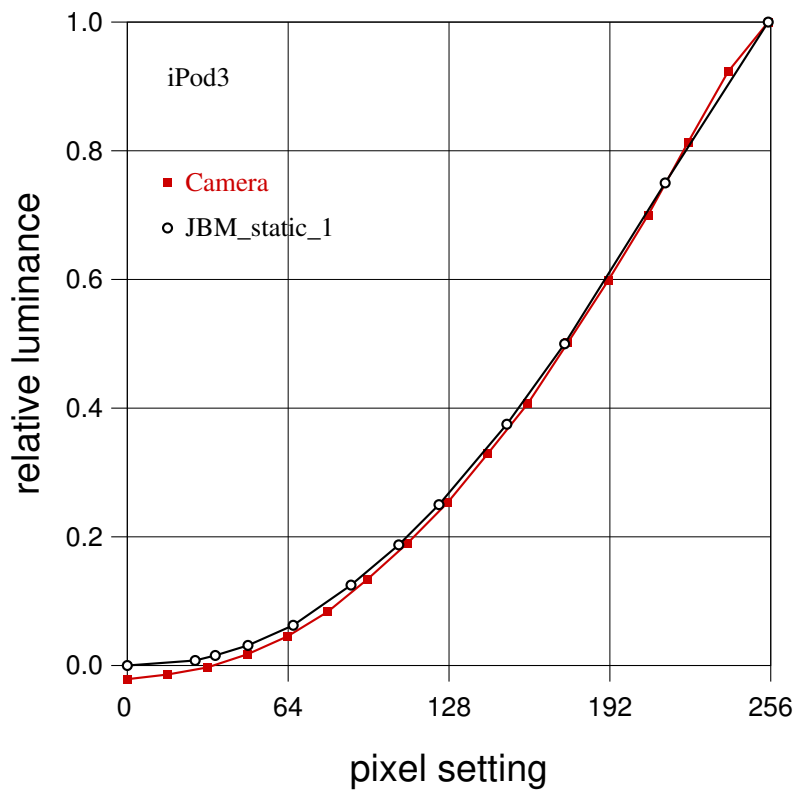

Figure 6: Comparison of device gamma estimates from psychophysical settings (black open symbols) and camera measurements (red filled symbols).

parameter, while the lower line shows the ascending order. We can see that the estimate peak sensitivity varies over a range of about $0.2 \log$ units, but appears to be stable after 6 swipes. Less variablity is seen in the estimage of the frequency of peak sensitivity. The subject in this experiment (the author) was wellmotivated, but was more concerned with speed over precision on the individual swipes, and so the variability from swipe-to-swipe probably represents motor system noise rather than a change in visual performance or criterion.

\section{Discussion}

While the proposed method offers a fast and convenient method for the estimation of the CSF, there are a few drawbacks. Perhaps most significant is the fact that there are no catch trials, so there is nothing to stop a subject that wanted to "cheat" from swiping in the invisible region. This problem might be addressed in part by presenting multiple variants of the pattern, in which the region of contrast space around the estimated threshold is expanded. A subject swiping in the invisible region would not be able to do this consistently in the absense of a visible reference, and so cheating might be detected by a high variance. This method would of course require additional swipes, losing some of the speed advantage.

\section{Summary}

We have presented a novel method for the rapid estimation of the human contrast sensitivity function (CSF), by having the subject perform a finger swipe over a contrast/frequency sweep image, using a mobile touch screen, allowing rapid assessment of vision using lightweight commodity hardware. The method enables rapid estimation of peak sensitivity with an accuracy of 0.1-0.2 log units.
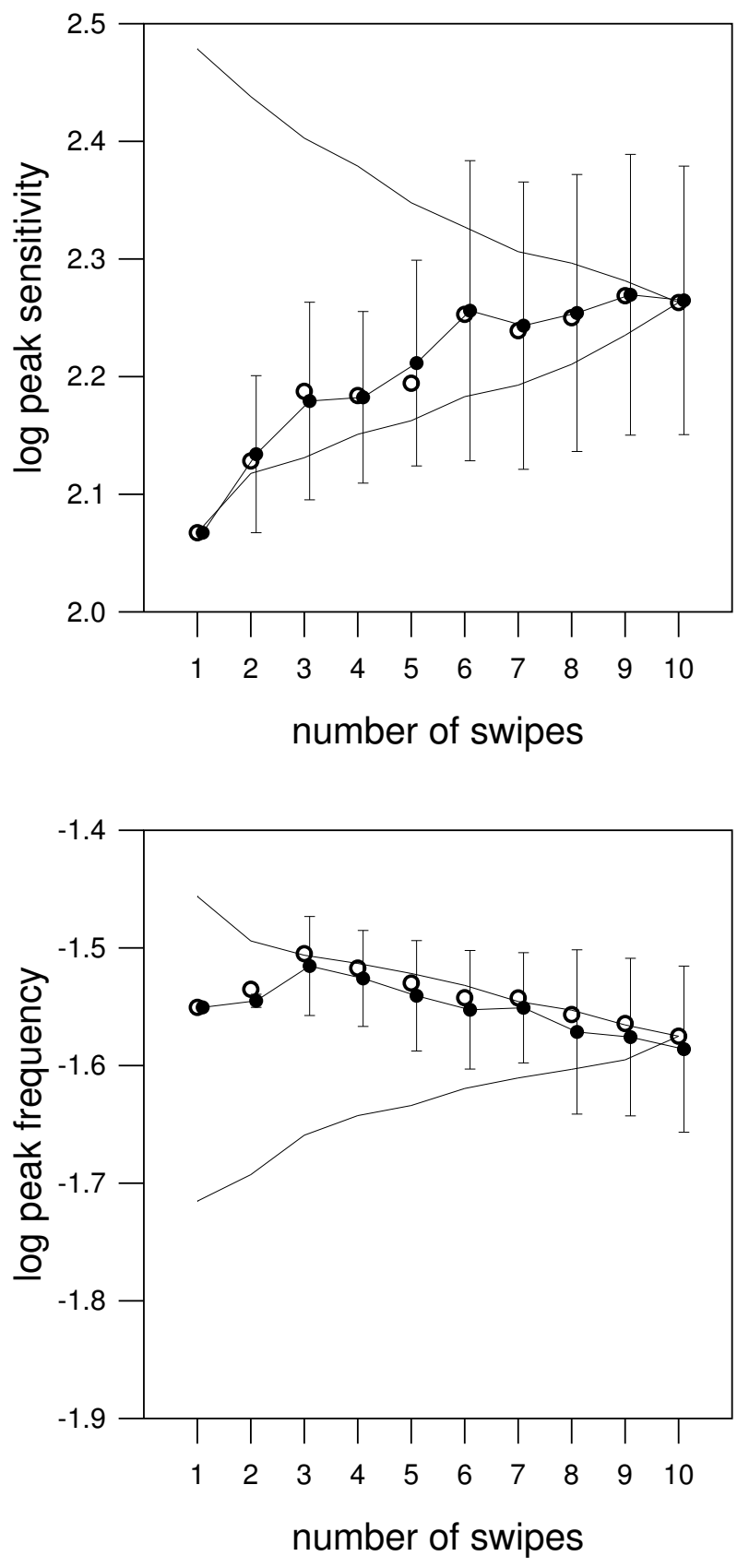

Figure 7: Evolution of estimated parameter values for 10 swipes. The thin lines represent the estimates obtained when the data are sorted in ascending and descending order.

\section{Acknowledgments}

Supported by the NASA Engineering Safety Center (NESC). Thanks to: John Robson for providing the history of the sweep grating and early research on contrast sensitivity; Jack Yellott for bringing the Washer and Roseberry paper [17] to my attention; and Michael Dorr for alerting me to the Uppsala Contrast Sensitivity Test [13]. 


\section{References}

[1] T. H. Mader, C. R. Gibson, A. F. Pass, L. A. Kramer, A. G. Lee, J. Fogarty, W. J. Tarver, J. P. Dervay, D. R. Hamilton, A. E. Sargsyan, J. L. Phillips, D. Tran, W. Lipsky, J. Choi, C. Stern, R. Kuyumjian, and J. D. Polk, "Optic disc edema, globe flattening, choroidal folds, and hyperopic shifts observed in astronauts after long-duration space flight," Ophthalmology, vol. 110, no. 10, pp. 2058-2069, 2011.

[2] J. A. Fogarty, C. Otto, E. Kerstman, C. Oubre, and J. Wu, "The visual impairment intracranial pressure summit report," NASA, Tech. Rep. TP-2011-216160, 2011.

[3] L. A. Kramer, A. E. Sargsyan, K. M. Hasan, J. D. Polk, and D. R. Hamilton, "Orbital and intracranial effects of microgravity: Findings at 3-T MR imaging," Radiology, vol. 263, no. 3, pp. 819-827, 2012.

[4] T. C. Wiener, "Space obstructive syndrome: Intracranial hypertension, intraocular pressure, and papilledema in space," Aviation, Space, and Environmental Medicine, vol. 83, no. 1, pp. 64-66, 2012.

[5] J. P. Berdahl, D. Y. Yu, and W. H. Morgan, "The translaminar pressure gradient in sustained zero gravity, idiopathic intracranial hypertension, and glaucoma," Medical Hypotheses, vol. 79, no. 6, pp. 719-724, 2012.

[6] K. Marshall-Bowman, M. R. Barratt, and C. R. Gibson, "Ophthalmic changes and increased intracranial pressure associated with long duration spaceflight: An emerging understanding," Acta Astronautica, vol. 87, pp. 77-87, 2013.

[7] E. S. Nelson, L. Mulugeta, and J. G. Myers, "Microgravity-induced fluid shift and ophthalmic changes," Life, vol. 4, no. 4, pp. 621-665, 2014.

[8] F. W. Campbell and J. G. Robson, "Application of fourier analysis to the visibility of gratings," J. Physiol., vol. 197, pp. 551-566, 1968.

[9] A. P. Ginsburg, "A new contrast sensitivity vision test chart," Am. J. Optom. Physiol. Opt., vol. 61, no. 6, pp. 403-407, 1984.

[10] D. G. Pelli, J. G. Robson, and A. J. Wilkins, "The design of a new letter chart for measuring contrast sensitivity," Clin. Vision Sci., vol. 2, no. 3, pp. 187-199, 1998.

[11] L. A. Lesmes, Z. L. Lu, J. Baek, and T. D. Albright, "Bayesian adaptive estimation of the constrast sensitivity function: The quick csf method," J. Vis., vol. 10, no. 3, pp. 17.1-17.21, 2010.

[12] M. Dorr, L. Lesmes, L. To, Z. L. Lu, and P. J. Bex, "High-precision psychophysics on the ipad," Perception, vol. 41, p. 98, 2012.

[13] L. D. Malmqvist and P. G. Soderberg, "The uppsala contrast sensitivity test (ucst): A fast strategy for clinical assessment of contrast sensitivity," in Proc. SPIE 8930, Ophthalmic Technologies XXIV, 2014, pp. 43-56.

[14] J. G. Robson, personal communcation, 2015.

[15] F. Ratliff, Mach Bands: Quantitative Studies on Neural Networks in the Retina. Holden Day, 1965.

[16] T. N. Cornsweet, Visual Perception. Academic Press, 1970.

[17] F. E. Washer and F. W. Roseberry, "New resolving power test chart," J. Opt. Soc. Am., vol. 41, no. 9, pp. 597-600, 1951.

[18] J. G. Robson, Contrast Sensitivity: One Hundred Years of Clinical Measurement. MIT Press, 1993.

[19] G. B. Arden, "The importance of measuring contrast sensitivity in cases of visual disturbance," British Journal of Ophthalmology, vol. 62, pp. 198-209, 1978.

[20] J. B. Mulligan and A. J. A. Jr., "Principled halftoning based on human vision models," in Proc. SPIE 1666, Human Vision, Visual Processing, and Digital Display III, 1992.

[21] J. B. Mulligan, "Digital halftoning methods for selectively partition- ing error into achromatic and chromatic channels," in Proc. SPIE 1249, Human Vision and Electronic Imaging: Models, Methods, and Applications, 1990, pp. 261-270.

[22] J. B. Mulligan and A. J. A. Jr., "Principled methods for color dithering based on models of the human visual system," in SID Intl. Symp. Dig. Tech. Papers, vol. 23, 1992, pp. 194-197.

[23] C. W. Tyler, "Colour bit-stealing to enhance the luminance resolution of digital displays on a single pixel basis," Spatial Vision, vol. 10, no. 4, pp. 369-377, 1997.

[24] J. B. Mulligan, "Methods for spatiotemporal dithering," in SID Intl. Symp. Dig. Tech. Papers, vol. 24, 1993, pp. 155-158.

[25] — "Application of temporal error diffusion to motion JPEG," in Proc. SPIE 3016, Human Vision and Electronic Imaging II, 1997, pp. 288-295.

[26] S. Anstis and P. Cavanagh, "A minimum motion technique for judging equiluminance," in Colour vision: psychophysics and physiology, J. B. Mollon and L. T. Sharpe, Eds. Academic Press, 1983, pp. $155-166$.

[27] J. B. Mulligan, "Presentation of calibrated images over the web," in Proc. SPIE 7240, Human Vision and Electronic Imaging XIV, 2009.

[28] M. Grossberg and S. Nayar, "Modeling the space of camera response functions," IEEE Trans. Pattern Anal. Mach. Intell., vol. 25, no. 11, pp. 1455-1467, 2003.

[29] P. Olczak and J. Tumblin, "Photometric camera calibration: Precise, labless, and automated with autolum," in ACM SIGGRAPH 2014 Posters, 2014, pp. 71-1.

[30] D. Marquardt, "An algorithm for least-squares estimation of nonlinear parameters," SIAM J. Appl. Math., vol. 11, pp. 431-441, 1963.

[31] M. I. A. Lourakis, "Sparse non-linear least squares optimization for geometric vision," in Proc. European Conference on Computer Vision, vol. 2, 2010, pp. 43-56.

[32] L. A. Lesmes and Z. L. Lu, "Methods and devices for rapid measurement of visual sensitivity," U.S. Patent 7938538, 2011.

\section{Author Biography}

Jeff Mulligan received the A.B. Degree in physics from Harvard University in 1980, and M.A. And Ph.D. Degrees in psychology from the University of California at San Diego in 1982 and 1986, respectively. In 1982 and 1983 he was a summer intern at AT\&T Bell Laboratories, in Murray Hill, New Jersey. Since then he has worked at NASA Ames Research Center, currently as a member of the Human Systems Integration division. He is a member of the Vision Sciences Society (VSS), the Optical Society (OSA), and the Society for Information Display (SID). 